\title{
Relativistic diffusive motion in random electromagnetic fields
}

\author{
Z. Haba \\ Institute of Theoretical Physics, University of Wroclaw, \\ 50-204 Wroclaw, Plac Maxa Borna 9, Poland \\ email:zhab@ift.uni.wroc.pl
}

November 13, 2018

\begin{abstract}
We show that the relativistic dynamics in a Gaussian random electromagnetic field can be approximated by the relativistic diffusion of Schay and Dudley. Lorentz invariant dynamics in the proper time leads to the diffusion in the proper time. The dynamics in the laboratory time gives the diffusive transport equation corresponding to the Jüttner equilibrium at the inverse temperature $\beta^{-1}=m c^{2}$. The diffusion constant is expressed by the field strength correlation function (Kubo's formula).
\end{abstract}

\section{Introduction}

The attempts to define a relativistic version of diffusion started a long time ago (see the reviews [1][2]). In [3][4] it has been shown that relativistic Markovian diffusion cannot be defined in the configuration space. It has been discovered by Schay [5] and Dudley [6] that Kramers phase space diffusion admits relativistic generalization. The generalization is unique under the assumption that the generator depends only on the phase space variables $(x, p)$ and that the particle mass does not change during the random evolution. It seems that the authors of ref.[7] unaware of the work of Schay and Dudley have introduced another definition of the relativistic diffusion which depends on an additional four-vector interpreted as a fluid velocity (without this four-vector the model would not be covariant). This approach is developed in [1][8]. In another approach [9]-[10] the authors following methods previously applied to the non-relativistic Langevin equation obtain the models of refs. [7][8] as well as the model of Schay and Dudley. We have discussed [11] drifts added to the relativistic diffusion of Schay and Dudley in such a way that an equilibrium is achieved and the detailed balance satisfied. This model has been independently discovered and studied in detail 
in [12]. The existence of an equilibrium which is the Jüttner distribution [13] has been also shown in the models of refs.[7][8] [9]. These diverse investigations did not show that there is a physical model realizing a particular version of the relativistic diffusion. The model of Schay and Dudley is appealing by its mathematical elegance (it has a generalization to arbitrary pseudoriemannian manifolds [14]). Nevertheless, it has been rejected in [1][8] as unphysical. In this paper we show that the relativistic diffusion of Schay and Dudley results from a dynamical model of a particle motion in a random electromagnetic field in the same way as the non-relativistic Brownian motion [15] can be derived from a non-relativistic dynamics in the electromagnetic field. Moreover, we show that a particular version of the relativistic Ornstein-Uhlenbeck process suggested in [11] and [12] (see also [10]) comes from an interaction with the relativistic random electromagnetic field.

In non-relativistic mechanics the diffusive dynamics can be derived from the dynamics in a random force [16][17][18][19] (these papers describe also the history of the problem with proper citations). The random force felt by a tracer particle can be understood as the force coming from a chaotic motion of other particles. The motion in a random electromagnetic field has been studied by physicists for a long time because of its relevance to astrophysics,plasma physics and high-energy physics [15] [20][21][22][23][24]. The notion of a random Liouville operator has been introduced by Kubo [25]. In his paper it has been shown that the Markov approximation to the random motion leads to the diffusion (for a rigorous proof of the non-relativistic diffusion see [16][17]). In view of the results obtained for non-relativistic dynamics it is suggestive to repeat the calculations in the relativistic case in order to see the appearance of the diffusive behaviour in a relativistic motion.

The plan of this paper is as follows. In sec.2 we define the relativistic dynamics of a massive particle in an electromagnetic field in the proper time and in the laboratory (coordinate) time. We move to a statistical description of classical dynamics in terms of the evolution of functions on the phase space generated by the Liouville first order differential operator. Such a formalism is a preparation for a unifying description of deterministic and random systems. In sec.3 we define a random Gaussian antisymmetric tensor field $F_{\mu \nu}$ which can be considered as an electromagnetic field whose randomness comes from random sources. To the best of author's knowledge the relativistic random electromagnetic field is constructed here for the first time. It could be considered as a regular(soft) version of quantum electromagnetic field when the short distance singularities of QED are ignored. We discuss an evolution of observables generated by (an adjoint of ) a random Lorentz invariant Liouville operator (sec.4) in the sense of Kubo [25]. We represent the evolution in a form suitable for averaging over the electromagnetic field. We refer to Kubo [25]-[26] for an argument that it is sufficient to calculate the expectation value of the square of the Liouville operator in order to determine the generator of the diffusion which results from the Markov approximation of the random dynamics. The calculation of an expectation value 
of the square of the Liouvile operator generating the proper time dynamics is performed in sec.5. It comes out that till the second order in the electromagnetic field the random dynamics coincides with the diffusive dynamics resulting from the diffusion generator of Schay and Dudley. This is the relativistic analog of the results derived for the non-relativistic motion in refs.[20][21][22]. It shows that for a particle motion in a random field the relativistic diffusion plays the same role as the classical Brownian motion.

We perform the same calculations in the laboratory time (sec.6). Now, we do not have the explicit Lorentz invariance of the Liouville operator. An average of the square of the Liouville operator over the electromagnetic field gives a surprise. There appears a first order Lorentz non-invariant term. We introduced such a drift in [11] as the friction determined by the Jüttner equilibrium distribution [13] through the detailed balance condition. The Jüttner distribution which comes from the random dynamics corresponds to the inverse temperature $\beta^{-1}=m c^{2}$ ( $m$ is the particle's mass). In the controversy concerning the relativistic analog of the Ornstein-Uhlenbeck process as discussed in [1][2][7] [8] the interaction with a random electromagnetic field selects the process determined by the detailed balance from the diffusion of Schay and Dudley.

\section{Relativistic dynamics}

The dynamics of a particle with mass $m$ in an external electromagnetic field neglecting its own electromagnetic field (produced by the current of a moving particle) is described by the explicitly Lorentz covariant four-vector equations $[27]$

$$
\begin{gathered}
\frac{d x^{\mu}}{d \tau}=\frac{1}{m c} p^{\mu}, \\
m c \frac{d p_{\mu}}{d \tau}=F_{\mu \nu} p^{\nu},
\end{gathered}
$$

The vectors are defined in the Minkowski space-time with the metric tensor $\eta^{\mu \nu}=(1,-1,-1,-1)$, the Greek index $\mu=0,1,2,3$. We shall also use Latin indices $j=1,2,3$. It follows from eq.(2) that

$$
\frac{d}{d \tau}\left(\eta^{\mu \nu} p_{\mu} p_{\nu}\right)=0 .
$$

Hence,

$$
p^{2}=\eta^{\mu \nu} p_{\mu} p_{\nu}=m^{2} c^{2},
$$

From eqs.(1) and (4) it follows that $\tau$ is the proper time

$$
d \tau^{2}=d x^{\mu} d x_{\mu} .
$$


We can eliminate $\tau$ from eqs.(1)-(2) in favor of $x^{0}$ (we call $x^{0}$ the laboratory time). Then, eqs.(1)-(2) read

$$
\begin{gathered}
\frac{d x^{k}}{d x^{0}}=\frac{1}{p_{0}} p^{k} \\
\frac{d p_{k}}{d x^{0}}=F_{k \nu} p^{\nu} p_{0}^{-1}
\end{gathered}
$$

$k=1,2,3$.

We move from a study of individual trajectories to functions $W$ (observables) on the phase space $(x, p)$. Then, $W$ evolves as

$$
\partial_{\tau} W=\frac{p^{\mu}}{m c} \frac{\partial W}{\partial x^{\mu}}-F_{j \nu} \frac{p^{\nu}}{m c} \frac{\partial W}{\partial p^{j}} .
$$

There is no derivative over $p^{0}$ in eq.(8) as $p^{0}$ is expressed by p. Knowing the evolution of $W$ we can obtain the trajectory $(x(\cdot), p(\cdot))$. It is useful to apply a statistical formulation of classical dynamics. We begin with a probability density $\Omega$ of the initial points $(x, p)$ in the phase space and determine its evolution in time $\tau$. We define an expectation value of the observable $W$ in a state (probability distribution) $\Omega$ as

$$
\Omega(W)=(\Omega, W)=\int d x d \mathbf{p} \Omega W
$$

Then, the adjoint evolution is

$$
\left(\Omega_{\tau}, W\right) \equiv\left(\Omega, W_{\tau}\right) .
$$

The initial probability distribution $\Omega$ in the phase space becomes a probability distribution of trajectories $(x(\cdot), p(\cdot))$ satisfying the equation

$$
\partial_{\tau} \Omega=-\frac{p^{\mu}}{m} \frac{\partial \Omega}{\partial x^{\mu}}+F_{j \nu} \frac{p^{\nu}}{m c} \frac{\partial \Omega}{\partial p^{j}} .
$$

The evolution of an observable $\psi$ in the laboratory time is determined by eqs. (6)-(7)

$$
\frac{\partial \psi}{\partial x^{0}}=\mathbf{p} p_{0}^{-1} \nabla_{\mathbf{x}} \psi+F_{j \nu} p^{\nu} p_{0}^{-1} \frac{\partial \psi}{\partial p^{j}} .
$$

By means of eq.(10) we can again define an evolution of the probability distribution. Such a statistical description is necessary when we consider the random dynamics because in such a case an individual trajectory is not an observable. Only averages over trajectories have a physical meaning. 


\section{Random electromagnetic fields}

We consider an antisymmetric tensor field $F$ which is covariant with respect to the Poincare group. We assume that an average $\langle\cdot\rangle$ over $F$ is defined which preserves the Poincare symmetry. This means that the two-point function defined by

$$
\left\langle F_{\mu \nu}(x) F_{\sigma \rho}\left(x^{\prime}\right)\right\rangle=G_{\mu \nu ; \sigma \rho}\left(x-x^{\prime}\right) .
$$

is a tensor depending only on $x-x^{\prime}$ with the following symmetry properties:

i) $G_{\mu \nu ; \sigma \rho}$ is symmetric under the exchange of indices $(\mu \nu ; x)$ and $\left(\sigma \rho ; x^{\prime}\right)$ and antisymmetric under the exchange $\mu \rightarrow \nu$ and $\sigma \rightarrow \rho$

We impose the first set of Maxwell equations (Bianchi identities) on the tensor field $F$

$$
\partial_{\mu} \epsilon^{\mu \nu \sigma \rho} F_{\sigma \rho}=0 .
$$

which imply an equation for the covariance (13)

ii)

$$
\partial_{\alpha} \epsilon^{\alpha \beta \mu \nu} G_{\mu \nu ; \sigma \rho}=0 .
$$

In Fourier transforms $(\tilde{F}(-k)=\overline{\tilde{F}}(k)$ because $F$ is real) eq.(13) reads

$$
\left\langle\tilde{F}_{\mu \nu}(k) \tilde{F}_{\sigma \rho}\left(k^{\prime}\right)\right\rangle=\tilde{G}_{\mu \nu ; \sigma \rho}(k) \delta\left(k-k^{\prime}\right),
$$

where $\tilde{G}_{\mu \nu ; \sigma \rho}(k)$ is a tensor which must be constructed from the vector $k_{\mu}$ and the fundamental four-dimensional tensors $\eta_{\mu \rho}$ and $\epsilon_{\mu \nu \rho \sigma}$. Hence, the tensor $\tilde{G}_{\mu \nu ; \sigma \rho}$ with the symmetries i) has the form

$$
\begin{aligned}
& \tilde{G}_{\mu \nu ; \sigma \rho}(k)=a_{1}(k)\left(\eta_{\mu \sigma} k_{\nu} k_{\rho}-\eta_{\mu \rho} k_{\nu} k_{\sigma}+\eta_{\nu \rho} k_{\mu} k_{\sigma}-\eta_{\nu \sigma} k_{\mu} k_{\rho}\right) \\
& +a_{2}(k)\left(\eta_{\mu \sigma} \eta_{\nu \rho}-\eta_{\mu \rho} \eta_{\nu \sigma}\right)+a_{3}(k) \epsilon_{\mu \nu \sigma \rho}
\end{aligned}
$$

where $a_{j}$ are scalars with respect to the Lorentz group. Eq.(15) after the Fourier transform reads

$$
k_{\alpha} \epsilon^{\alpha \beta \mu \nu} \tilde{G}_{\mu \nu ; \sigma \rho}(k)=0 .
$$

It follows from eqs.(17) and (18) that $a_{1}$ can be an arbitrary function of $k^{2}$ and $a_{2}=0$. From eq.(18) we obtain an equation for $a_{3}$

$$
k_{\alpha} \epsilon^{\alpha \beta \mu \nu} \epsilon_{\mu \nu \sigma \rho} a_{3}(k)=0 .
$$

Hence, $a_{3}(k)=0$ if $k \neq 0$ (i.e. $a_{3}=K \delta(k)$ ). In the configuration space the admissible $a_{3}$ term would lead to a constant term ( $K$ is a constant)

$$
G_{\mu \nu ; \sigma \rho}^{\epsilon}=K \epsilon_{\mu \nu \sigma \rho} .
$$

So far we did not make any assumption concerning the question where the average value (13) comes from. As an example, it could come from a time average. It may result from a certain probability distribution of the currents

$$
\partial^{\mu} F_{\mu \nu}=J_{\nu}
$$


or the electromagnetic field becomes random as a result of a passage through a random medium. In the latter cases there exists a probability measure determining the distribution of $F_{\mu \nu}$. It is known [28] that a necessary condition for an existence of such a measure is

$$
\left\langle\left(\int d x F_{\mu \nu}(x) f^{\mu \nu}(x)\right)^{2}\right\rangle=\int d x d x^{\prime} f^{\mu \nu}(x) f^{\sigma \rho}\left(x^{\prime}\right) G_{\mu \nu ; \sigma \rho}\left(x-x^{\prime}\right) \geq 0
$$

for arbitrary test-functions $f^{\mu \nu}$ (satisfying some regularity and growth conditions [28]). The condition (22) is also sufficient for an existence of a random Gaussian field $F$.

The positivity condition (22) excludes the $\epsilon$-term (20) because the quadratic form

$$
\int d x d x^{\prime} f^{\mu \nu}(x) f^{\sigma \rho}\left(x^{\prime}\right) \epsilon_{\mu \nu \sigma \rho}
$$

cannot be positive for arbitrary test-functions $f^{\mu \nu}$. It follows that only the $a_{1}$ term, which is a function of $k^{2}$, remains in eq.(17). Hence,

$$
\left\langle F_{\mu \nu}(x) F_{\sigma \rho}\left(x^{\prime}\right)\right\rangle=-D_{\mu \nu ; \sigma \rho} G\left(x-x^{\prime}\right),
$$

where

$$
D_{\mu \nu ; \sigma \rho}=-\eta_{\mu \sigma} \partial_{\nu} \partial_{\rho}+\eta_{\mu \rho} \partial_{\nu} \partial_{\sigma}-\eta_{\nu \rho} \partial_{\sigma} \partial_{\mu}+\eta_{\nu \sigma} \partial_{\mu} \partial_{\rho}
$$

and $G$ is a function depending only on $\left(x-x^{\prime}\right)^{2}$.

There remains to explore the positivity condition (22). We show that under the assumptions i)-ii) there exists a random antisymmetric tensor field with the two-point correlation function (23) if and only if the Fourier transform $\tilde{G}$ of the function $G$ in eq.(23) satisfies the condition

iii)

$$
\tilde{G}(k) \geq 0
$$

and $\tilde{G}(k)=0$ if $k^{2}<0$.

We prove that the inequality (22) follows from the condition iii). With the two-point correlation function (23) eq.(22) reads

$$
\begin{aligned}
& \int d x d x^{\prime} f^{\mu \nu}(x) f^{\sigma \rho}\left(x^{\prime}\right) G_{\mu \nu ; \sigma \rho}\left(x-x^{\prime}\right) \\
& =-\int d k \tilde{f}^{\mu \nu}(-k)\left(\eta_{\mu \sigma} k_{\nu} k_{\rho}-\eta_{\mu \rho} k_{\nu} k_{\sigma}+\eta_{\nu \rho} k_{\mu} k_{\sigma}-\eta_{\nu \sigma} k_{\mu} k_{\rho}\right) \tilde{f} \sigma \rho \tilde{G}(k) \\
& =\int d k \overline{g^{j}(k)} g^{j}(k) \tilde{G}(k)-\int d k \overline{k_{j} \tilde{f}^{0 j}} k_{l} \tilde{f}^{0 l}(k) \tilde{G}(k),
\end{aligned}
$$

where

$$
g^{j}=k_{0} \tilde{f}^{0 j}+k_{l} \tilde{f}^{l j} .
$$

Expressing $k_{j} \tilde{f}^{0 j}$ by $k_{j} g^{j}$ we obtain

$$
\begin{aligned}
& \int d x d x^{\prime} f^{\mu \nu}(x) f^{\sigma \rho}\left(x^{\prime}\right) G_{\mu \nu ; \sigma \rho}\left(x-x^{\prime}\right)=\int d k \tilde{G}(k) \overline{g^{j}} g^{j}-\int d k \tilde{G}(k) k_{0}^{-2}\left|g^{j} k_{j}\right|^{2} \\
& \geq \int d k \tilde{G}(k) k_{0}^{-2} \overline{g^{j}} g^{j}\left(k_{0}^{2}-\mathbf{k}^{2}\right) \geq 0,
\end{aligned}
$$


if (25) and the condition iii) are satisfied.

Remarks:

1.Note that there is a function $M_{\nu \sigma \rho}$ such that

$$
\left\langle\partial^{\mu} F_{\mu \nu}(x) F_{\sigma \rho}\left(x^{\prime}\right)\right\rangle=\partial^{\mu} \partial_{\mu} M_{\nu \sigma \rho}\left(x-x^{\prime}\right) .
$$

We treat this consequence of eq.(23) as a weak form of the second set of Maxwell equations. The condition that the electromagnetic field has no source $(J=0)$ can now be imposed by the requirement that $M$ satisfies the wave equation .

2. If the source-less Maxwell equations (21) are satisfied then

$$
\tilde{G}(k)=\delta\left(k^{2}\right)
$$

(eq.(29), with $k_{0} \geq 0$, is satisfied for the vacuum two-point function in quantum field theory of the free electromagnetic field [29]). However, the two-point function (23) determined by its Fourier transform (29) is singular. The singularity would appear in the diffusion equation as a singularity of the diffusion coefficients. We do not impose $J=0$ in eq.(21) in order to work with more regular electromagnetic fields.

It follows from eqs.(23)-(24) that

$$
D_{\mu \nu ; \sigma \rho} G=\eta_{\mu \sigma} G_{\nu \rho}-\eta_{\mu \rho} G_{\nu \sigma}+\eta_{\nu \rho} G_{\sigma \mu}-\eta_{\nu \sigma} G_{\mu \rho}
$$

with

$$
G_{\mu \nu}(x) \equiv \partial_{\mu} \partial_{\nu} G \equiv \eta_{\mu \nu} h_{1}(x)+x_{\mu} x_{\nu} h_{2}(x) .
$$

Here, $G(x) \equiv g(u)$ with $u=x^{2}, h_{1}(x)=2 g^{\prime}(u)$ and $h_{2}(x)=4 g^{\prime \prime}(u)$. The functions $h_{j}(x)$ depend only on $x^{2}=x_{\mu} x^{\mu}$.

If in a model of an electromagnetic field the scalar $G$ is a function of some other vectors (in addition to $x$ ) then $\mathrm{G}$ is a function of scalars built from these vectors. In such a case the formula (31) contains more terms. As a consequence, the diffusion discussed in subsequent sections would also depend on the additional vectors.

\section{Random evolution}

In this section we discuss a solution of the evolution equations (8) and (11). We are going to represent the solution in such a form that an averaging over the electromagnetic field can be performed. The differential equations (8) and (11) for the evolution of functions $W$ on the phase space are of the form

$$
\partial_{s} W_{s}=(X+Y) W_{s},
$$

where $Y$ is a random first order differential operator and $X$ is the free evolution. For the evolution (8) in the proper time

$$
X=\frac{1}{m c} p^{\mu} \partial_{\mu} .
$$


Let

$$
Y(s)=\exp (-s X) Y \exp (s X) .
$$

Then, the solution of eq.(32) can be expressed as

$$
W_{t}=\exp (t X) W_{t}^{I}
$$

where

$$
\partial_{s} W_{s}^{I}=Y(s) W_{s}^{I},
$$

(this is the interaction picture well-known from quantum mechanics). We can solve eq.(36) by iteration. The iteration till the second order reads

$$
\begin{aligned}
& W_{t}^{I}=W_{0}+\int_{0}^{t} d s Y(s) W_{s}^{I}+\int_{0}^{t} d s \int_{0}^{s} d s^{\prime} Y(s) Y\left(s^{\prime}\right) W_{s^{\prime}}^{I} \\
& =W_{0}+\int_{0}^{t} d s Y(s) W_{s}^{I}+\frac{1}{2} \int_{0}^{t} d s \int_{0}^{s} d s^{\prime}\left(Y(s) Y\left(s^{\prime}\right)+Y\left(s^{\prime}\right) Y(s)+\left[Y(s), Y\left(s^{\prime}\right)\right]\right) W_{s^{\prime}}^{I} .
\end{aligned}
$$

We can expand the solution (37) in an infinite series. We assume that the initial condition $W$ for eq.(32) is independent of the electromagnetic field. Then, the expectation values of the products of $Y(s)$ appearing in the series can be calculated because the correlation functions of the Gaussian random field $F$ are determined by the two-point function (23). In the form of a path-ordered integral the solution has the form

$$
W_{t}^{I}=T\left(\exp \left(\int_{0}^{t} d s Y(s)\right)\right) W_{0}
$$

The diffusion limit applied in secs.5-6 relies on the approximation [25][26]

$$
\left\langle T\left(\exp \left(\int_{0}^{t} d s Y(s)\right)\right)\right\rangle=\exp \left(\frac{1}{2} \int_{0}^{t} d s \int_{0}^{s} d s^{\prime}\left\langle\left(Y(s) Y\left(s^{\prime}\right)+Y\left(s^{\prime}\right) Y(s)\right)\right\rangle\right) .
$$

If $\left[Y(s), Y\left(s^{\prime}\right)\right]=0$ and $Y$ is a linear function of Gaussian variables then eq.(39) is exact. In our case the operator $Y(s)$ (generating the Liouville dynamics) is an antihermition operator in $L^{2}\left(\frac{d \mathbf{p}}{p_{0}} d x\right)$. Then, the anticommutator $Y(s) Y\left(s^{\prime}\right)+Y\left(s^{\prime}\right) Y(s)$ is a hermition operator whereas $\left[Y(s), Y\left(s^{\prime}\right)\right]$ is antihermition. We neglect the commutator in eqs.(37) and (39) corresponding to the unitary Liouville evolution and restrict ourselves to the pure diffusion.

\section{The expectation value of the proper time evo- lution}

The observable time evolution results from an averaging over fluctuations of the electromagnetic field. The fluctuations of the electromagnetic field make the dynamics chaotic. The particles' trajectories become random. In this section we apply the approach of Kubo [25] [26] in order to obtain a diffusion as a Markov 
approximation to random dynamics. The result involves an approximation of the Liouville evolution of functions on the phase space by an evolution generated by second order differential operators. In this formulation it is sufficient to calculate an expectation value of the square of the Liouville operator in order to determine the generator of the diffusion. We consider the proper time evolution (1)-(2) first. In general, we could split the Liouville operator $Y^{t o t}=Y^{e x}+Y$, where $Y^{e x}$ is the Liouville operator corresponding to an external deterministic electromagnetic field and $Y$ describes the random part. The $Y^{e x}$ part could be added to the final result. We restrict our discussion to the random Liouville operator (34). Taking as $Y$ the second term in eq.(8) and $X$ from eq.(33) we calculate $Y(s)(34)$

$$
Y(s)=F_{j \nu}\left(x-\frac{s}{m c} p\right) p^{\nu} \frac{\partial}{\partial p^{j}} .
$$

We apply the covariance (30)-(31) (with $x^{\mu} \simeq \frac{s}{m c} p^{\mu}$ from eq.(40)) to calculate the expectation value appearing in eq.(39)

$$
\begin{aligned}
& \frac{1}{2} \int_{0}^{\tau} d s \int_{0}^{s} d s^{\prime}\left\langle\left(Y(s) Y\left(s^{\prime}\right)+Y\left(s^{\prime}\right) Y(s)\right)\right\rangle=(m c)^{-2} \int_{0}^{\tau} d s \int_{0}^{s} d s^{\prime} \\
& \left(\eta_{j l}\left(\eta_{\nu \rho} H_{1}\left(s-s^{\prime}\right)+m^{-2} c^{-2} p_{\nu} p_{\rho} H\left(s-s^{\prime}\right)\right)\right. \\
& -\eta_{j \rho}\left(\eta_{\nu l} H_{1}\left(s-s^{\prime}\right)+m^{-2} c^{-2} p_{\nu} p_{l} H\left(s-s^{\prime}\right)\right) \\
& +\eta_{\nu \rho}\left(\eta_{j l} H_{1}\left(s-s^{\prime}\right)+m^{-2} c^{-2} p_{j} p_{l} H\left(s-s^{\prime}\right)\right) \\
& \left.-\eta_{\nu l}\left(\eta_{j \rho} H_{1}\left(s-s^{\prime}\right)+m^{-2} c^{-2} p_{j} p_{\rho} H\left(s-s^{\prime}\right)\right)\right) p^{\nu} \frac{\partial}{\partial p^{j}} p^{\rho} \frac{\partial}{\partial p^{l}},
\end{aligned}
$$

where

$$
H_{1}\left(s-s^{\prime}\right)=h_{1}\left(\left(s-s^{\prime}\right)^{2}\right)=2 g^{\prime}(u)
$$

and

$$
H\left(s-s^{\prime}\right)=\left(s-s^{\prime}\right)^{2} h_{2}\left(\left(s-s^{\prime}\right)^{2}\right)=4 u g^{\prime \prime}(u) .
$$

where $u=\left(s-s^{\prime}\right)^{2}, h_{j}(x)$ (defined after eq.(31)in terms of $G\left(x^{2}\right) \equiv g\left(\sqrt{x^{2}}\right)$ ) being functions of $x^{2}$ depend only on $s-s^{\prime}$ because after a calculation of the two-point function (13) of $F\left(x-\frac{s}{m c} p\right)$ and $F\left(x-\frac{s^{\prime}}{m c} p\right)$ we obtain

$$
h_{j}\left(\left(\left(s-s^{\prime}\right) p(m c)^{-1}\right)^{2}\right)=h_{j}\left(\left(s-s^{\prime}\right)^{2}\right) .
$$

The explicit calculations in eq.(41) give

$$
\frac{1}{2} \int_{0}^{\tau} d s \int_{0}^{s} d s^{\prime}\left\langle\left(Y(s) Y\left(s^{\prime}\right)+Y\left(s^{\prime}\right) Y(s)\right)\right\rangle=(m c)^{-2} \int_{0}^{\tau} d s \int_{0}^{s} d s^{\prime}\left(2 H_{1}\left(s-s^{\prime}\right)+H\left(s-s^{\prime}\right)\right) \triangle_{H}^{m},
$$

where

$$
\triangle_{H}^{m}=\left(\delta^{j l} m^{2} c^{2}+p^{j} p^{l}\right) \frac{\partial}{\partial p^{l}} \frac{\partial}{\partial p^{j}}+3 p^{l} \frac{\partial}{\partial p^{l}}
$$

is the generator of the relativistic diffusion of Schay [5] and Dudley [6]. In the approximation (39) (neglecting the non-commutativity) the result of an average over a random electromagnetic field is the relativistic diffusion with the time 
transformed by the function standing in front of $\triangle_{H}^{m}$ in eq.(42). Note that $\triangle_{H}^{m}$ is self-adjoint in $L^{2}\left(d \mathbf{p} p_{0}^{-1}\right)$ similarly as its non-relativistic analog (generator of Krammers diffusion) is self-adjoint in $L^{2}(d \mathbf{p})$.

\section{Random evolution in laboratory time}

Eqs.(1)-(2) are equivalent to eqs.(6)-(7). Both sides of eqs.(1)-(2) are fourvectors transforming linearly under the Lorentz group. This property does not hold true for eqs.(6)-(7). The equivalence between the description in terms of the proper time and coordinate time may be lost if we perform non-linear transformations such as the averaging over random fields. For this reason we calculate the expectation values of the square of the laboratory time Liouville generator (12) again. In the laboratory time the free evolution is determined by

$$
X=p_{0}^{-1} \mathbf{p} \nabla_{\mathbf{x}} .
$$

Then from eqs.(12) and (34)

$$
Y(s)=F_{l \mu}\left(\mathbf{x}-s p_{0}^{-1} \mathbf{p}, s\right) p^{\mu} p_{0}^{-1} \frac{\partial}{\partial p^{l}} .
$$

For the calculation of the expectation values of $Y$ (45) we must make the replacements in formulas of sec.5

$$
\begin{aligned}
p & \rightarrow p_{0}^{-1} \mathbf{p}, \\
\mathbf{x} & \rightarrow p_{0}^{-1} \mathbf{p} s
\end{aligned}
$$

and $x_{0}-x_{0}^{\prime}=s-s^{\prime}$. So that

$$
\left(x-x^{\prime}\right)^{2}=p_{0}^{-2} m^{2} c^{2}\left(s-s^{\prime}\right)^{2}
$$

as the argument of the functions $h_{j}$ in eq.(41). From eq.(46) we can see that the transition from the proper time evolution to the laboratory time evolution involves $\mathbf{p} \rightarrow \mathbf{p} p_{0}^{-1}$. This is equivalent to $s \rightarrow s m c p_{0}^{-1}$ in the functions $h_{j}$ when we calculate the expectation value (41). Changing the time integration variables $s \rightarrow s m c p_{0}^{-1}$ in eq.(41) we obtain (we write $x_{0}=c t$ )

$$
\begin{aligned}
& \frac{1}{2} \int_{0}^{c t} d s \int_{0}^{s} d s^{\prime}\left\langle\left(Y(s) Y\left(s^{\prime}\right)+Y\left(s^{\prime}\right) Y(s)\right)\right\rangle \\
& =\int_{0}^{t m c^{2} p_{0}^{-1}} d s \int_{0}^{s} d s^{\prime}\left(\eta_{j l}\left(\eta_{\nu \rho} H_{1}\left(s-s^{\prime}\right)+m^{-2} c^{-2} p_{\nu} p_{\rho} H\left(s-s^{\prime}\right)\right)\right. \\
& -\eta_{j \rho}\left(\eta_{\nu l} H_{1}\left(\left(s-s^{\prime}\right)+m^{-2} c^{-2} p_{\nu} p_{l} H\left(s-s^{\prime}\right)\right)\right. \\
& +\eta_{\nu \rho}\left(\eta_{j l} H_{1}\left(\left(s-s^{\prime}\right)+m^{-2} c^{-2} p_{j} p_{l} H\left(s-s^{\prime}\right)\right)\right. \\
& \left.-\eta_{\nu l}\left(\eta_{j \rho} H_{1}\left(s-s^{\prime}\right)+m^{-2} c^{-2} p_{j} p_{\rho} H\left(s-s^{\prime}\right)\right)\right) p_{0} p^{\nu} \frac{\partial}{\partial p^{j}} p^{\rho} p_{0}^{-1} \frac{\partial}{\partial p^{l}} .
\end{aligned}
$$


We have an additional term $D_{1}$ (coming from $p^{j}$ differentiation of $p_{0}^{-1}$ on the rhs of eq.(47)) in comparison to the rhs of eq.(41)

$$
D_{1}=-p_{0}^{-1} p^{\nu} p^{j} \frac{\partial}{\partial p^{l}}
$$

This term after a contraction with the tensors in eq.(47) gives the result

$$
\frac{1}{2} \int_{0}^{c t} d s \int_{0}^{s} d s^{\prime}\left\langle\left(Y(s) Y\left(s^{\prime}\right)+Y\left(s^{\prime}\right) Y(s)\right)\right\rangle=\int_{0}^{t m c^{2} p_{0}^{-1}} d s \int_{0}^{s} d s^{\prime}\left(2 H_{1}\left(s-s^{\prime}\right)+H\left(s-s^{\prime}\right)\right) \triangle_{\beta}
$$

with

$$
\triangle_{\beta}=\triangle_{H}^{m}-p_{0} p^{j} \frac{\partial}{\partial p^{j}}
$$

where the last term in eq.(50) is the friction introduced in [11]. $\triangle_{\beta}$ generates a diffusion which equilibrates to the Jüttner distribution with the inverse temperature $\beta^{-1}=m c^{2}$. We obtain the Jüttner equilibrium distribution [13] $\Phi_{E}=\exp \left(-\beta c p_{0}\right)$ from the requirement

$$
\triangle_{\beta}^{*} \Phi_{E}=0,
$$

where the adjoint is in $L^{2}(d \mathbf{p})$ (see eq.(9)).

It is surprising that the proper time dynamics (1)-(2) and the laboratory time dynamics (6)-(7) after an average over the electromagnetic field give inequivalent results. The probability distribution $\Omega$ of the random dynamics in the laboratory time tends to the Jüttner equilibrium distribution $\Phi_{E}$ when time goes to infinity. The covariant dynamics of sec.5 has no limit when the proper time or the laboratory time go to infinity. It is easy to comprehend the difference from the technical point of view as the Liouville generator in laboratory time dynamics has the $p_{0}^{-1}$ factor which leads to the extra term (48). However, some mysteries remain concerning the question why equivalent dynamics have different diffusive limits.

We assume that the functions $H_{1}$ and $H$ decay fast for $s \neq s^{\prime}$. We approximate the $s^{\prime}$-integral appearing in eqs.(42) and (49) as follows

$$
\int_{0}^{s} d s^{\prime}\left(2 H_{1}\left(s-s^{\prime}\right)+H\left(s-s^{\prime}\right)\right) \simeq \int_{0}^{\infty} d s^{\prime}\left(2 H_{1}\left(s^{\prime}\right)+H\left(s^{\prime}\right)\right)=\frac{\kappa^{2}}{2},
$$

where $\kappa^{2}$ is the diffusion constant of ref.[11]. We apply the formulae below eq.(41), expressing $H_{1}$ and $H$ in terms of $G(x)$ to calculate

$$
\kappa^{2}=2 \int_{0}^{\infty} d u u^{-\frac{1}{2}} g^{\prime}(u)
$$

,where $G(x) \equiv g(u)$ with $u=x^{2}$.

The Markov approximation must be performed also for higher order terms in the expansion (37) in order to justify the formula (39). The approximation has 
been discussed first by Kubo [25]-[26] (the expression of the diffusion constant (51) and (52) by the correlation function of the forces $F_{\mu \nu}$ defining the functions $h_{j}$ is known as the Kubo formula). Summarizing the results (42) and (49) we have in the Markov approximation

$$
\partial_{\tau} W_{\tau}^{I}=\frac{\kappa^{2}}{2} \triangle_{H}^{m} W_{\tau}^{I}
$$

When we define $W$ as in eq.(35) then $W$ satisfies the equation

$$
\partial_{\tau} W_{\tau}=p^{\mu} \frac{\partial}{\partial x^{\mu}} W_{\tau}+\frac{\kappa^{2}}{2} \triangle_{H}^{m} W_{\tau} .
$$

In the laboratory time the corresponding equations read

$$
p_{0} \frac{\partial}{\partial x_{0}} W_{x_{0}}^{I}=\frac{\kappa^{2}}{2} \triangle_{\beta} W_{x_{0}}^{I} .
$$

and

$$
p^{\mu} \frac{\partial}{\partial x^{\mu}} W=\frac{\kappa^{2}}{2} \triangle_{\beta} W
$$

\section{Summary and outlook}

We have been studying a model of particle relativistic dynamics in a random electromagnetic field showing diffusive behaviour. The aim was to find which of the models of the relativistic diffusion encountered in the literature is realized in this physical model. For this reason we have calculated an expectation value of the square of the Liouville operator which defines the generator of the diffusion. In the case of the dynamics in proper time, as the result of the calculations, we obtain the generator of the diffusion of Schay and Dudley; a mathematical model discovered a long time ago but disregarded by most physicists. We repeat the calculations for the dynamics in the laboratory time. As a result we obtain the generator of the proper time diffusion plus a drift term which moves the probability distribution to the equilibrium (the Jüttner equilibrium distribution at $\beta^{-1}=m c^{2}$ ). Comparing with the non-relativistic dynamics in the electromagnetic field we can see that the diffusive dynamics in the proper time can be treated as a relativistic analog of the Brownian motion whereas the dynamics in the laboratory time is analogous to the one of the Ornstein-Uhlenbeck

process. It follows that the diffusion of Schay and Dudley should be treated as a starting point for an investigation of the diffusive motion of relativistic systems in the same way as the Brownian motion (in Krammers version) serves for a construction of other non-relativistic diffusions in physics [15] as well as in mathematics.

Some extensions of our formulation can be suggested. Applying the MartinSiggia-Rose formalism [30][18][19] one could obtain a functional measure (defined on trajectories) corresponding to the motion in a random electromagnetic 
field. The exact formula for the functional measure can be applied for further investigations of the random motion beyond the Markov approximation. It could also be used for an inquiry of the meaning of the proper time dynamics in random relativistic systems and the relation to laboratory time dynamics. The equilibration to the Jüttner distribution (expected on physical grounds) resulting from the generator (50) may indicate a deeper mathematical mechanism (in the path integral formalism) leading to the relativistic version of the OrnsteinUhlenbeck process. In another direction, we can assume a dependence of the two-point function (13) on some other tensors (e.g. a vector describing a moving frame for theories at finite temperature). In such a case the formalism does not change. We can calculate the dependence of the resulting diffusion (which can be treated as a perturbation of the one studied here) on the additional variables. Then, astrophysical applications of the relativistic diffusion require a general pseudoriemannian metric (as in [14]). The formulation of secs.2-6 can be extended to general relativity. A local Markov approximation leading to the diffusion on a manifold is still possible. However, a global approximation of the relativistic dynamics on a manifold by the diffusion of ref.[14] may encounter some difficulties.

\section{References}

[1] C. Chevalier and F. Debbasch, AIP Conf.Proc.913,42(2007)

[2] J. Dunkel and P. Hänggi,Phys.Rep.471,1(2009)

[3] J. Lopuszanski, Acta Phys.Pol.12,87(1953)

[4] R. Hakim, J.Math.Phys.9,1805(1968)

[5] G.Schay,PhD thesis,Princeton University,1961

[6] R.Dudley, Arkiv for Matematik,6,241(1965)

[7] F. Debbasch, K. Mallik and J.P. Rivet, Journ.Stat.Phys.88,945(1997)

[8] C. Chevalier and F. Debbasch, J.Math.Phys.49,043303(2008)

[9] J.Dunkel, P. Talkner and P. Hänggi, Phys.Rev.D75,043001(2007)

[10] J. Dunkel and P. Hänggi, Phys.Rev.E72,036106(2005)

[11] Z. Haba, Phys.Rev.E79,021128(2009)

[12] J.A. Alcantara and S. Calogero, Kinetic and Related Models, 4,401(2011)

[13] F. Jüttner, Ann.Phys.(Leipzig)34,856(1911) 
[14] J. Franchi and Y. Le Jan, Commun.Pure and Appl.Math. 60,187(2007)

[15] S. Chandrasekhar, Rev.Mod.Phys.15,1(1943)

[16] H. Kesten and G.C. Papanicolaou, Commun.Math.Phys.78,19(1980)

[17] T.Komorowski and L. Ryzhik, Commun.Math.Phys.263,277(2006)

[18] N. Lebedev, P. Maass and S. Feng, Phys.Rev.Lett, 74,1895(1995)

[19] L. Golubovic, S. Feng and Fan-An Zeng, Phys. Rev. Lett.67,2115(1991)

[20] J.R. Jokpii,Astrphys.J.146,480(1966)

[21] P.A. Sturrock, Phys.Rev.141,186(1966)

[22] D.E.Hall and P.A. Sturrock, Phys.Fluids 10,2620(1967)

[23] W.B. Thompson and J. Hubbard, Rev.Mod.Phys.32,714(1960)

[24] B.Svetitsky, Phys.Rev.D37,2484(1988)

[25] R. Kubo, J.Math.Phys.4,174(1962)

[26] R. Kubo, M. Toda and N. Hashitsume, Statistical Physics II.Nonequilibrium Statistical Mechanics, Springer, Berlin, 1985

[27] L.D. Landau and E.M. Lifshits, Field Theory, Pergamon Press, New York, 1981

[28] I.M. Gelfand and N.Y. Vilenkin, Generalized Functions.Vol.IV, Academic Press,New York, 1964

[29] S.S. Schweber, An Introduction to Relativistic Quatum Field Theory, Row,Peterson and Co, Evanston,1961

[30] P.C. Martin, E.D. Siggia and H.A. Rose, Phys.Rev.A8,423(1973) 